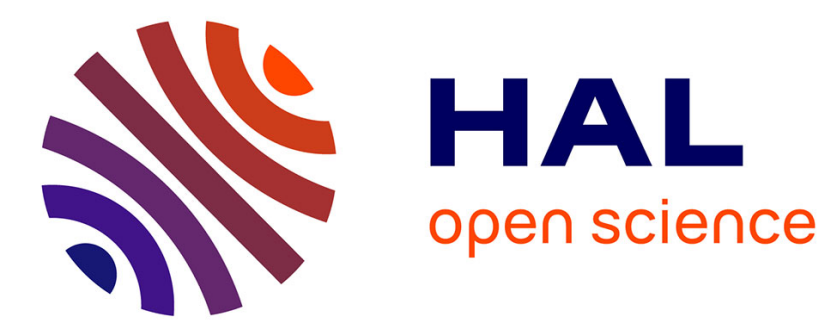

\title{
Une approche micro-macro pour le suivi de fissure avec enrichissement local
}

Pierre-Alain Guidault, Olivier Allix, Laurent Champaney, Jean-Phillippe

Navarro

\section{- To cite this version:}

Pierre-Alain Guidault, Olivier Allix, Laurent Champaney, Jean-Phillippe Navarro. Une approche micro-macro pour le suivi de fissure avec enrichissement local. 7e colloque national en calcul des structures, CSMA, May 2005, Giens, France. hal-01812939

\section{HAL Id: hal-01812939 \\ https://hal.science/hal-01812939}

Submitted on 12 Jun 2018

HAL is a multi-disciplinary open access archive for the deposit and dissemination of scientific research documents, whether they are published or not. The documents may come from teaching and research institutions in France or abroad, or from public or private research centers.
L'archive ouverte pluridisciplinaire HAL, est destinée au dépôt et à la diffusion de documents scientifiques de niveau recherche, publiés ou non, émanant des établissements d'enseignement et de recherche français ou étrangers, des laboratoires publics ou privés. 


\title{
Une approche micro-macro pour le suivi de fissure avec enrichissement local
}

\author{
Pierre-Alain Guidault* - Olivier Allix* - Laurent Champaney ${ }^{* *}$ \\ - Jean-Phillippe Navarro ${ }^{* * *}$ \\ * Laboratoire de Mécanique et Technologie, ENS de Cachan \\ CNRS/Université Pierre et Marie Curie \\ 61 avenue du Président Wilson, F-94235 Cachan cedex \\ \{guidault,allix\}@lmt.ens-cachan.fr \\ ** Laboratoire d'Etudes Mécaniques des Assemblages, \\ Université de Versailles/Saint-Quentin-en-Yvelines/CNRS \\ 45 avenue des Etats-Unis, F-78035 Versailles Cedex \\ *** Dassault Aviation \\ 78 quai Marcel Dassault, Cedex 300, F-92552 Saint-Cloud Cedex
}

RÉSUMÉ. Dans ce papier, une stratégie de calcul multiéchelle pour le suivi de fissure est présentée. Cette stratégie a pour objectif de séparer les effets locaux des effets globaux afin de garder un maillage macro inchangé au cours de la propagation tout en permettant l'utilisation d'une description fine uniquement où cela est nécessaire. Deux points sont discutés : le premier est le choix d'une échelle macro rendant compte de l'effet macro de la fissure; le second est l'utilisation d'une décomposition du domaine en sous-structures et interfaces permettant de limiter l'utilisation d'une échelle fine uniquement dans le voisinage de la fissure. L'intégration de la X-FEM comme méthode d'enrichissement local pour décrire la fissure est aussi présentée. ABSTRACT. In this paper, a multiscale strategy for the analysis of crack propagation is presented. The purposes of this strategy are, first, to separate the local effects from the global effects in order to keep a macromesh unchanged during the crack's propagation and, second, to enable one to use a proper fine-scale description only where it is required. Two aspects are discussed: the first is the choice of the macroscale in order to include the macroeffect of a crack; the second is the use of a decomposition of the domain into substructures and interfaces in order to limit the use of the refined scale only around the crack. The integration of the X-FEM as a local enrichment method for the description of a crack is also presented.

MOTS-CLÉS : Stratégie multiéchelle, propagation de fissure, X-FEM, homogénéisation, enrichissement macro, enrichissement micro.

KEYWORDS: Multiscale strategy, crack propagation, X-FEM, homogenization, macroenrichment, microenrichment. 


\section{Introduction}

Le calcul des structures avec fissuration est aujourd'hui revisité par des techniques émergentes comme l'approche des fortes discontinuités (SDA) introduite par Oliver [OLI 02]. D'autres approches telles que la méthode des éléments finis étendus (XFEM) [DAU 00] et la méthode des éléments finis généralisés (GFEM) [STR 01], se basent sur la méthode de la partition de l'unité (PUM) introduite par Melenk et Babuška en 1996 [MEL 96]. Le maillage n'ayant pas besoin d'être conforme à la géométrie de la fissure, les opérations de maillage sont grandement simplifiées. Cependant, ces techniques n'incorporent pas complètement l'aspect multiéchelle induit par la localisation des déformations dans la zone fissurée. Aussi, la nécessité d'avoir un maillage fin en pointe de fissure conduit à un remaillage global de la structure au cours de la propagation.

Pour pallier cette difficulté liée au remaillage, la stratégie développée en collaboration avec Dassault Aviation a pour objectifs, premièrement, de séparer le traitement des effets locaux des effets globaux afin de ne pas changer le maillage «macro» au cours de la propagation de la fissure et, deuxièmement, de pouvoir utiliser une description à l'échelle fine seulement dans les zones pertinentes (Figure 1). La démarche

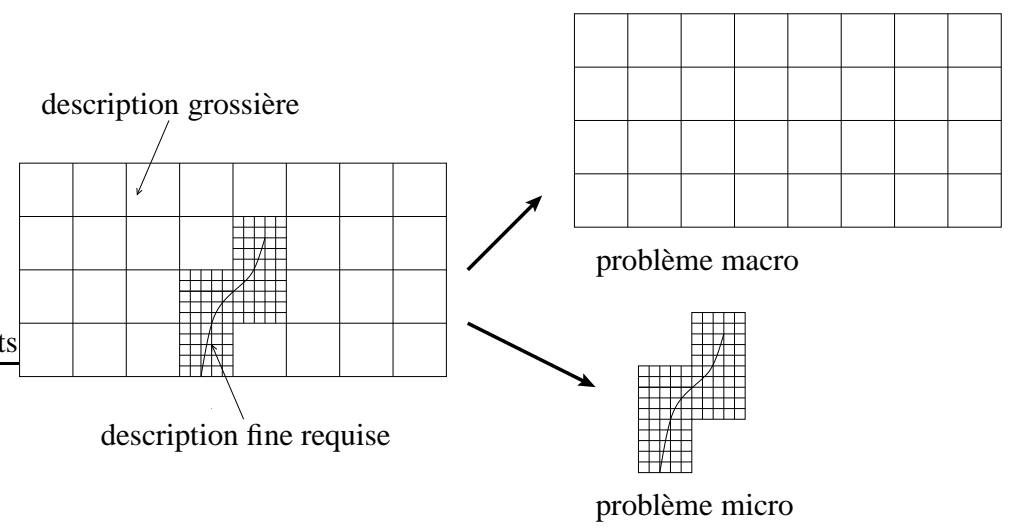

Figure 1. Structure fissurée : problème macro et problème micro

ainsi envisagée met en synergie deux techniques [GUI 04]. La première est issue de travaux menés au LMT-Cachan concernant la stratégie micro-macro [LAD 01] initialement dédiée à l'étude de structures hétérogènes et bâtie sur une technique d'homogénéisation. La deuxième technique basée sur la PUM est utilisée pour représenter correctement la solution locale à l'échelle micro. L'introduction des fonctions d'enrichissement est réalisée au moyen de la X-FEM.

Une fissure ayant une influence à la fois au niveau local et au niveau global, la question de la description de la cinématique et des efforts aux deux niveaux est alors soulevée. Dans l'approche micro-macro appliquée au suivi de fissure trois principaux 
aspects ont ainsi été étudiés. Le premier est l'utilisation d'une interface permettant la mise en place d'une description fine dans le voisinage de la fissure et traitant le problème d'incompatibilité de maillage de façon naturelle. Le second est le choix de l'échelle macro et de ses enrichissements pour inclure l'effet macro d'une fissure. Enfin, le troisième point porte sur l'intégration de la X-FEM au niveau micro. Il est montré que, dans le cadre de l'approche multiéchelle utilisée, celle-ci ne pose pas de difficultés particulières.

\section{L'approche multiéchelle pour le suivi de fissure}

L'approche micro-macro s'articule autour de trois points fondamentaux :

- Un partitionnement de la structure en sous-structures et interfaces

Une sous-structure $E$ est soumise à l'action de son environnement (les interfaces voisines) qui se traduit par une distribution d'effort $\underline{F}_{E}$ et une distribution de déplacement $\underline{W}_{E}$. Ce partitionnement confère le caractère mixte à cette méthode de décomposition de domaine.

- Un aspect multiéchelle introduit au niveau des interfaces

Contrairement à la plupart des approches multiéchelles, la séparation entre les échelles ne se fait qu' au niveau des interfaces. Les quantités d'interface s'écrivent ainsi comme la somme d'une partie macro et d'une partie micro. Ainsi, $\underline{F}_{E}=\underline{F}_{E}^{M}+\underline{F}_{E}^{m}$.

- Une vérification partielle des conditions de transmission

Les efforts macro d'interface doivent systématiquement vérifier les conditions de transmission a priori. L'espace associé est noté $\mathcal{F}_{a d}^{M}$.

L'algorithme itératif LATIN [LAD 99] est alors utilisé pour résoudre. Deux groupes d'équations $A_{d}$ et $\Gamma$ permettant de séparer les difficultés sont ainsi constitués :

$$
\begin{aligned}
& \begin{array}{l||l} 
& -1 \text { l'admissibilité statique de }\left(\sigma_{E}, \underline{F}_{E}\right), \forall M \underline{M} \in \Omega_{E} \\
A_{d} & - \text { l'admissibilité cinématique de }\left(\epsilon_{E}, \underline{W} E\right), \forall \underline{M} \in \Omega_{E} \\
& - \text { l'admissibilité de } \bigcup_{E \in \mathbf{E}}\left\{\underline{F}_{E}^{M}\right\} \in \mathcal{F}_{a d}^{M}
\end{array}
\end{aligned}
$$

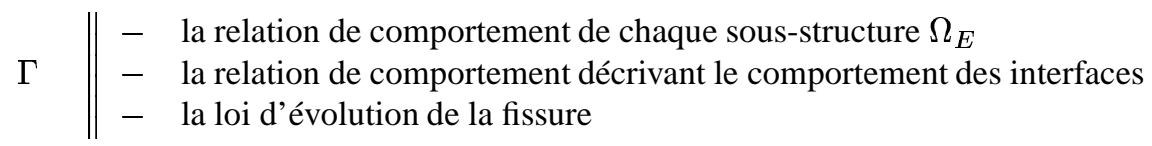

$\Gamma$ regroupe les équations locales éventuellement non-linéaires et $A_{d}$, les équations linéaires éventuellement globales. La stratégie consiste à chercher une solution qui vérifie alternativement les équations de $A_{d}$ puis celles de $\Gamma$ par le biais de deux directions de recherche rendant le problème bien posé. L'admissibilité de $\bigcup_{E \in \mathbf{E}}\left\{\underline{F}_{E}^{M}\right\} \in \mathcal{F}_{a d}^{M}$ conduit à un problème macro portant sur les efforts macro $\underline{F}^{M}$.

Concernant le suivi de fissure, nous proposons un partitionnement basé sur un maillage éléments finis grossier (Figure 1). Chaque élément représente une sousstructure. Un raffinement peut alors être requis pour certaines sous-structures. La ques- 
tion d'incompatibilité de maillage se pose alors. De même, une fissure pouvant traverser une interface, l'introduction d'une discontinuité en déplacement doit être étudiée. L'interface permet de traiter efficacement ces difficultés.

\section{Description de l'interface et séparation des échelles}

Les quantités d'interface s'écrivent sous la forme $\underline{F}=\underline{F}^{M}+\underline{F}^{m}$ et $\underline{W}=\underline{W}^{M}+$ $\underline{W}^{m}$. Les parties macro sont obtenues par un projecteur $\Pi$ tel que :

$$
\int_{\Gamma_{E E^{\prime}}} \underline{F} \cdot \underline{W} d S=\int_{\Gamma_{E E^{\prime}}} \underline{F}^{M} \cdot \underline{W}^{M} d S+\int_{\Gamma_{E E^{\prime}}} \underline{F}^{m} \cdot \underline{W}^{m} d S
$$

On a alors : $\underline{F}^{M}=\Pi(\underline{F})$ et $\underline{F}^{m}=(i d-\Pi)(\underline{F})$. Il en est de même pour $\underline{W}$.

L'incompatibilité de maillage entre deux sous-structures $E$ et $E^{\prime}$, l'une raffinée et l'autre non, est traitée par le raccord [GUI 04] :

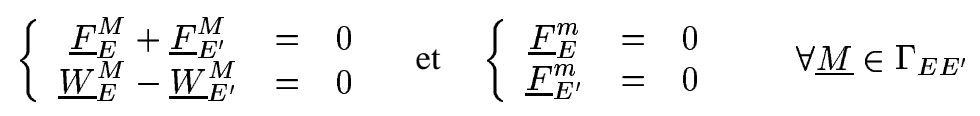

Un tel raccord consiste alors à recoller les quantités d'efforts généralisés, résultante et moment, comme une application du principe de Saint-Venant.

Afin de ne pas changer la structure du problème macro, la discontinuité en déplacement n'est introduite qu'au niveau micro. Les quantités macro $\underline{F}^{M}$ et $\underline{W}^{M}$ sont ainsi continues et, par conséquence, les quantités micro sont discontinues [GUI 04].

\section{Enrichissement de la base macro}

Le choix du projecteur macro est arbitraire et propre à chaque interface. Il est donc possible de choisir un projecteur qui extrait non seulement la partie affine mais aussi la partie cubique pour les interfaces situées dans la zone fissurée (Figure 2). Un tel enrichissement local de la base macro permet alors de mieux décrire l'effet macro de la fissure et d'améliorer la convergence de la stratégie (Figure 3).

\section{Implantation de la $X$-FEM au niveau micro}

Afin de s'affranchir des difficultés de maillage au niveau micro, une technique d'enrichissement locale comme la X-FEM pour représenter la fissure est utilisée. Le multiéchelle n'étant introduit qu'au niveau des interfaces, il est possible d'enrichir l'approximation du champ de déplacement $\underline{u}_{E}$ sur une sous-structure $E$ suivant la technique illustrée dans [STO 01].

$$
\underline{u}_{E_{h}}(\underline{x})=\sum_{i \in N} \varphi_{i}(\underline{x}) \underline{u}_{i}+\sum_{i \in N_{d}} \varphi_{i}(\underline{x}) H(\underline{x}) \underline{a}_{i}+\sum_{i \in N_{p}} \varphi_{i}(\underline{x})\left(\sum_{j=1}^{4} F_{j}(\underline{x}) \underline{b}_{i}^{j}\right)
$$




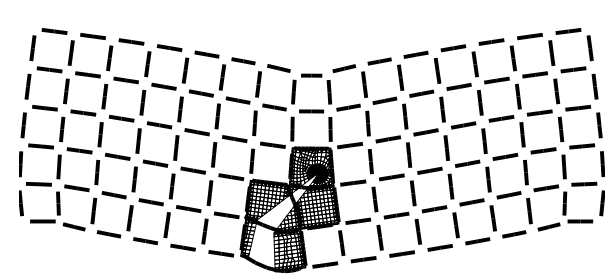

(a) Déformée et déplacements macros

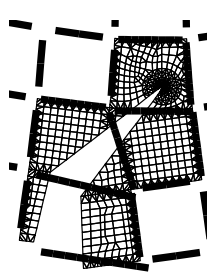

(b) linéaire

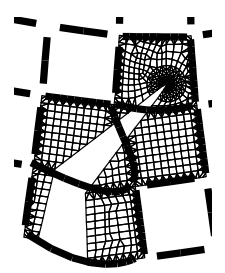

(c) cubique

Figure 2. Déformée et déplacement macro $\underline{W}_{E}^{M}$ (traits épais continus) avec une base macro linéaire pour chaque interface 2(b) et un enrichissement macro cubique local 2(c) pour les quatre interfaces situées dans la zone raffinée

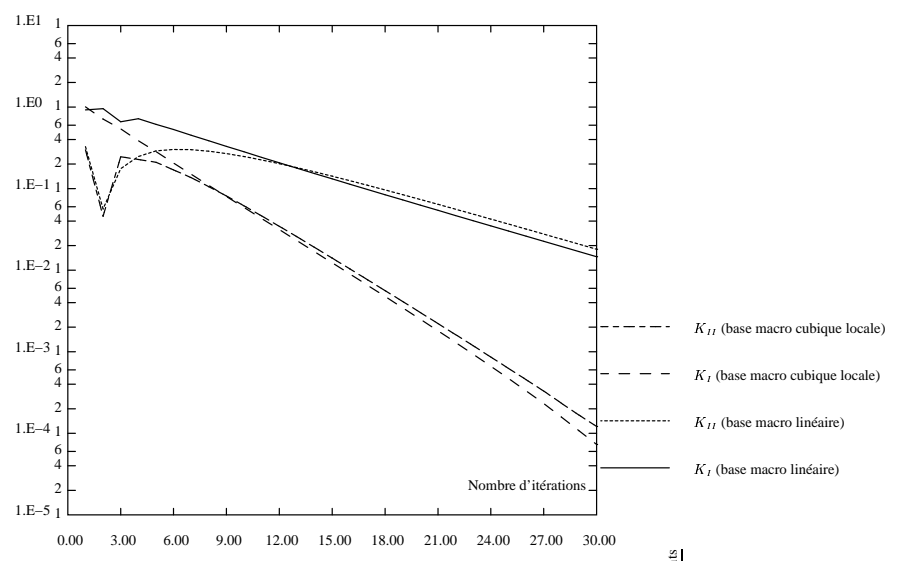

Figure 3. Convergence de l'erreur relative des facteurs d'intensité de contrainte $K_{I}$ et $K_{I I}$ avec comme référence la solution à convergence de l'algorithme

où $H$ et $\left\{F_{j}\right\}$ représentent les fonctions d'enrichissement habituelles de discontinuité et de solution asymptotique en pointe de fissure. On peut alors réécrire $\underline{u}_{E_{h}}$ sous la forme $\underline{u}_{E_{h}}(\underline{x})=\sum_{i \in N} \tilde{\varphi}_{i}(\underline{x}) \underline{d}_{E_{i}}$ où les fonctions $\tilde{\varphi}_{i}$ représentent sans distinction les fonctions de forme éléments finis classiques $\varphi_{i}$ et les fonctions de forme enrichies $H \varphi_{i}$ et $F_{j} \varphi_{i}$.

Le projecteur macro $\Pi$ est défini avant même toute discrétisation. La définition des quantités macro et micro restent donc inchangée. Toute la difficulté réside alors dans l'admissibilité cinématique du champ $\underline{u}_{E}$ et du déplacement bord $\underline{W}$ :

$$
\forall \underline{F} \in \mathcal{F}_{E E^{\prime}}, \quad\left(\underline{F},\left(\underline{u}_{E}-\underline{W}\right)\right)_{\Gamma_{E E^{\prime}}}=0
$$


Les quantités d'interface sont discrétisées et également enrichies par $H$ sous la forme $\underline{F}=\sum_{i \in N_{\Gamma}} \psi_{i}(\underline{x}) \underline{f}_{i}+\sum_{i \in N_{\Gamma_{d}}} \psi_{i}(\underline{x}) H(\underline{x}) \underline{a}_{\Gamma_{i}}=\sum_{i \in N_{\Gamma}} \tilde{\psi}_{i}(\underline{x}) \underline{F}_{i}$. Après discrétisation de [3], on obtient: $\left[N_{E E^{\prime}}\right]\left[B_{E E^{\prime}}\right]\left[d_{E}\right]=\left[M_{E E^{\prime}}\right]\left[W_{E E^{\prime}}\right]$ où $\left[B_{E E^{\prime}}\right]$ est l'opérateur de restriction booléen des inconnues nodales $\left[d_{E}\right]$ au bord $\Gamma_{E E^{\prime}},\left[N_{E E^{\prime}}\right]_{i j}=$ $\left(\tilde{\psi}_{i}, \tilde{\varphi}_{\left.j\right|_{\Gamma_{E E^{\prime}}}}\right)_{\Gamma_{E E^{\prime}}}$ et $\left[M_{E E^{\prime}}\right]_{i j}=\left(\tilde{\psi}_{i}, \tilde{\psi}_{\left.j\right|_{\Gamma_{E E^{\prime}}}}\right)_{\Gamma_{E E^{\prime}}}$. $\left[W_{E E^{\prime}}\right]$ est le vecteur des inconnues nodales en déplacement de $\Gamma_{E E^{\prime}}$. Le choix de la discrétisation des quantités d'interfaces avec l'enrichissement $H$ est actuellement en cours d'étude.

\section{Conclusion}

Une stratégie de calcul multiéchelle pour le suivi de fissure a été présentée. Cette stratégie permet de séparer les effets macro des effets micro de la fissure afin de garder un maillage macro inchangé au cours de la propagation tout en permettant l'utilisation d'une description fine uniquement où cela est nécessaire. L'intégration de la X-FEM pour décrire la fissure au niveau micro pour s'affranchir des difficultés de remaillage à l'échelle fine, s'inscrit aisément dans le cadre multiéchelle que nous avons présenté. Les travaux actuels porte sur la simulation de la propagation de fissure en fatigue.

\section{Bibliographie}

[DAU 00] Daux C., Moës N., Dolbow J., Sukumar N., Belytschko T., « Arbitrary branched and intersecting cracks with the extended finite element method », International Journal for Numerical Methods in Engineering, vol. 48, 2000, p. 1741-1760.

[GUi 04] Guidault P.-A., Allix O., Champaney L., Navarro J.-P., « A micro-macro approach for crack propagation with local enrichment », Proceedings of the Seventh International Conference on Computational Structures Technology, Lisbon, Portugal, 2004.

[LAD 99] LADEVÈzE P., Nonlinear Computational Structural Mechanics - New Approaches and non-Incremental Methods of Calculation, Springer Verlag, 1999.

[LAD 01] LADEVÈze P., LoIseAu O., Dureisseix D., « A micro-macro and parallel computational strategy for highly heterogeneous structures », International Journal for Numerical Methods in Engineering, vol. 52, $\mathrm{n}^{\circ}$ 1-2, 2001, p. 121-138.

[MEL 96] Melenk J., BABUŠKA I., « The Partition of Unity Finite Element Method : Basic Theory and Applications », Computer Methods in Applied Mechanics and Engineering, vol. 139, 1996, p. 289-314.

[Oli 02] Oliver J., Huespe A. E., Pulido M., Chaves E., « From continuum mechanics to fracture mechanics : the strong discontinuity approach », Engineering Fracture Mechanics, vol. 69, 2002, p. 113-136.

[STO 01] Stolarska M., Chopp D. L., Mö̈s N., Belytschko T., « Modelling Crack Growth by Level Sets and the Extended Finite Element Method », International Journal for Numerical Methods in Engineering, vol. 51, $\mathrm{n}^{\circ}$ 8, 2001, p. 943-960.

[STR 01] Strouboulis T., Copps K., BABušKa I., « The generalized finite element method », Computer Methods in Applied Mechanics and Engineering, vol. 190, 2001, p. 40814193. 